\title{
Error Concealment of H.264 Encoded Video through a Hybrid Scheme
}

\author{
Chinmay Kumar Nayak \\ Defence Research and \\ Development Organisation \\ India \\ chinmay@iitb.ac.in \\ Jayalakshmi Surendran * \\ Vidyalankar Institute of \\ Technology, Mumbai \\ India \\ jayalakshmi.surendran@gmail.com \\ S. N. Merchant \\ Indian Institute of Technology \\ Bombay \\ India \\ merchant@ee.iitb.ac.in \\ U. B. Desai \\ Indian Institute of Technology \\ Hyderabad \\ India \\ ubdesai@ee.iitb.ac.in \\ Sugata Sanyal \\ Tata Institute of Fundamental \\ Research \\ Mumbai, India \\ sanyal@tifr.res.in
}

\begin{abstract}
A hybrid error concealment scheme for H.264/AVC that uses the concept of watermarking, Overlapping Boundary Matching Algorithm (OBMA) and Projection Onto Convex Sets (POCS) is proposed in this paper. The advantage of this scheme is that, watermarking is performed during the encoding process itself. The specific features of both intra coded and inter coded frames are exploited for this purpose. The proposed method chooses watermarking or OBMA or POCS or a combination of them selectively for error concealment which minimizes computational complexity. Simulations are performed to show that the proposed method works well with those videos which have abruptly moving blocks.
\end{abstract}

\section{Categories and Subject Descriptors}

H.4 [Information Systems Applications]: Miscellaneous; D.2.8 [Software Engineering]: Metrics - complexity measures, performance measures

\section{General Terms}

H.264/AVC, Error Concealment

\section{Keywords}

Watermarking, Projection Onto Convex Sets, Overlapping Boundary Matching Algorithm

\section{INTRODUCTION}

${ }^{*}$ Corresponding Author.

Permission to make digital or hard copies of all or part of this work for personal or classroom use is granted without fee provided that copies are not made or distributed for profit or commercial advantage and that copies bear this notice and the full citation on the first page. To copy otherwise, to republish, to post on servers or to redistribute to lists, requires prior specific permission and/or a fee.

MEDES'10 October 26-29, 2010, Bangkok, Thailand

Copyright 2010 ACM 978-1-4503-0047-6/10/10 ...\$10.00.
Data transmitted over channels are vulnerable to transmission errors. Imperfect transmission of block coded images and video results in loss of blocks. Error correction, error control and error concealment techniques have been developed for reducing these visual artifacts [11]. Error concealment techniques reduce image distortions through post processing at the decoder side. Hence error concealment techniques do not have access to the original information and are usually based on estimation and interpolation procedures that do not require additional information from the encoder.

Digital watermarking is basically a method of inserting some content into the original data which can be later recovered to prove authentication or copyright. Of late it has also been proposed for error concealment of images and video [1]. In this case some important information derived from the image itself is chosen as the watermark and is retrieved at the decoder as in the case of a blind watermarking scheme and used for concealing errors. For the purpose of error concealment of H.264/AVC, we make use of three different methods in this paper. One is based on digital watermarking where the average values of the lost blocks are restored from the hidden data. The second method used for concealment of errors is OBMA. OBMA is employed in those blocks which have no motion or uniform motion. The third method is POCS which is a computationally expensive method unless combined with another method of error concealment. So this method is used only in the last stage of concealment where error is very predominant due to loss of edge information.

POCS [9] utilizes correlated edge information from local neighborhood in images and intra frames of video to restore missing blocks. It is an iterative algorithm satisfying spatial domain and spectral domain constraints. Thus POCS method preserves edge, but is computationally complex and non real time as it requires much iteration to extrapolate missing pixels from the available correctly received surrounding blocks.

OBMA finds the motion vector that yields the best overlapping between the external boundaries of the lost macroblocks in both the present frame and the reference frame [4]. Watermarking ensures that each block is replaced by 
its average value. But this has the disadvantage of high blockiness effect as the number of error blocks increases. This can be improved through POCS by recovering coefficients extrapolated from the neighboring correctly received blocks. POCS is an efficient algorithm to maintain edge continuity. Moreover, it gives superior concealment for blocks in smooth regions by exploiting the local texture information. POCS being a computationally expensive method, is applied after replacing error blocks with their average values through watermarking based method. This reduces the number of iterations for POCS. Most of the erroneous blocks are concealed using watermarking based method. So a low complexity error measure is worked out to select blocks on which POCS will be applied. This ensures better performance with reduced complexity. Peak Signal to Noise Ratio and Structural Similarity (SSIM) [12] are chosen as two performance indices for evaluating this method.

\section{WATERMARKING BASED ERROR CON- CEALMENT}

This section gives a detailed description of the watermark embedding and extraction process. The method conceals the error in the damaged blocks using the hidden data in the image [6]. For this purpose the entire image is given equal importance and there is no segregation as Region Of Interest and Region Of Background. The hidden information in the image basically contains the average values of the image. A wavelet based algorithm is used for this purpose and the gray level values of the approximate band is hidden in two selected sub-bands of the image. Before hiding these values directly, they are encrypted using a pseudo-random sequence generated by using a key. So only a person who has the key can decrypt the hidden information for concealment purpose.

\section{IMPLEMENTATION OF POCS}

Watermarking based error concealment gives good performance when the number of blocks in error is less. As more number of blocks are lost during transmission, it becomes difficult to retrieve the correct copy of the embedded data. This affects the quality of the error concealed image. Thus, with a large number of error blocks, it is better to combine another method for further improvement in the image quality. In POCS, lost image blocks are restored by extending edges present in the surrounding neighborhood so that they pass through the missing block. If no edges are present in the surrounding neighborhood, the lost block is restored by a smoothing process.

\section{ERROR CONCEALMENT OF H.264/AVC}

It has been shown that watermark embedding prior to encoding along with POCS works well for error concealment of images [7]. This concept along with some modification has been efficiently introduced for error concealment of H.264/AVC in this section. Video frames in any codec will be either intra-coded or inter-coded. In order to enhance compression, H.264/AVC performs predictive coding in both I and $\mathrm{P}$ frames [8], [10], [5]. The data to be transmitted corresponding to each frame thus consists of information of predicted frame as well as the difference frame. In this particular codec, IntegerDCT is used for encoding
[8], [2]. The approximate band is embedded into the transmitted difference frame and is used for error concealment in case of block errors.

As mentioned above, each frame is encoded through predictive coding. In the case of intra coded frames, each macro block is predicted from the previously encoded pixels in the neighborhood of the same frame. The residual frame is formed by finding the difference between the predicted frame and the original frame. Thus, both predicted frame and the residual frame are transmitted. On the contrary, for inter coded frames, motion vectors are estimated with respect to the reference frame. The residual frame is formed by finding the difference between the original and the motion compensated predicted frame. Here motion vector information and residual frame are transmitted. Irrespective of the prediction, whether intra coded or inter coded, H.264/AVC encodes the residual information through IntegerDCT or IntDCT. The watermark bits for each frame will be formed as explained in [6]. A three level wavelet transformation is performed on the original frames and the approximate band is scaled with scaling factor. These values are then converted to binary array. This forms the data to be embedded into the residual data during the encoding process.

\subsection{Watermark Embedding}

The watermark embedding steps on a residual frame is shown in Figure 1.

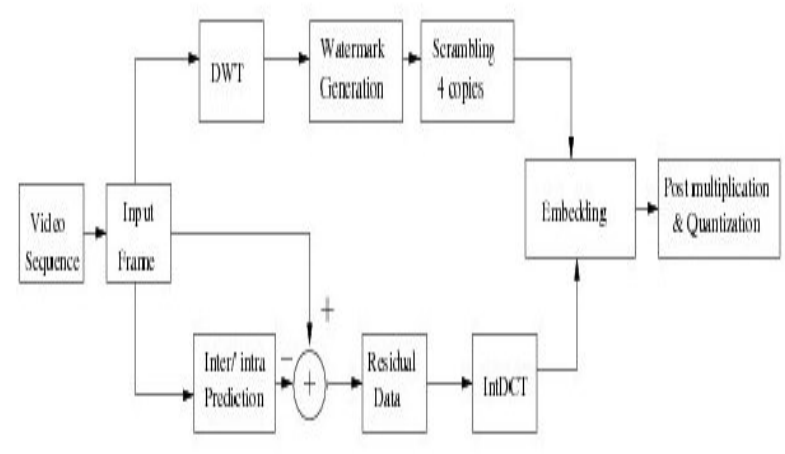

Figure 1: Watermark embedding on residual frame

IntDCT is operated on $4 \times 4$ blocks of residual data obtained after motion compensated prediction or intra prediction. The forward transform is given by Equation 1

$$
Y=\left(C_{f} X C_{f}^{T}\right) \otimes E_{f}
$$

Here $\mathrm{X}$ is the $4 \times 4$ matrix to be transformed in the residual frame and $\mathrm{Y}$ is the transformed matrix.

$$
C_{f}=\left(\begin{array}{cccc}
1 & 1 & 1 & 1 \\
2 & 1 & -1 & -2 \\
1 & -1 & -1 & 1 \\
1 & -2 & 2 & -1
\end{array}\right)
$$




$$
E_{f}=\left(\begin{array}{cccc}
a^{2} & \frac{a b}{2} & a^{2} & \frac{a b}{2} \\
\frac{a b}{2} & \frac{b^{2}}{4} & \frac{a b}{2} & \frac{b^{2}}{4} \\
a^{2} & \frac{a b}{2} & a^{2} & \frac{a b}{2} \\
\frac{a b}{2} & \frac{b^{2}}{4} & \frac{a b}{2} & \frac{b^{2}}{4}
\end{array}\right)
$$

Here $a=\frac{1}{2}, b=\sqrt{\frac{2}{5}}$ and $\otimes$ denotes point by point multiplication. IntDCT has identical compression performance as DCT, but has the added advantage of operation without loss of decoding accuracy. $C_{f} X C_{f}^{T}$ gives the forward IntDCT of the matrix $X$. The inverse transform of $Y$ is given by Equation 2.

$$
\begin{gathered}
X=C_{i}^{T}\left(Y \otimes E_{i}\right) C_{i} \\
C_{i}=\left(\begin{array}{cccc}
1 & 1 & 1 & \frac{1}{2} \\
1 & \frac{1}{2} & -1 & -1 \\
1 & \frac{-1}{2} & -1 & 1 \\
1 & -1 & 1 & \frac{-1}{2}
\end{array}\right) \\
E_{i}=\left(\begin{array}{cccc}
a^{2} & a b & a^{2} & a b \\
a b & b^{2} & a b & b^{2} \\
a^{2} & a b & a^{2} & a b \\
a b & b^{2} & a b & b^{2}
\end{array}\right)
\end{gathered}
$$

Once $4 \times 4$ IntDCT is performed, out of the 16 coefficients in each of the $4 \times 4$ matrix, 8 coefficients are selected as shown in Figure 2. The watermark bits generated are embedded
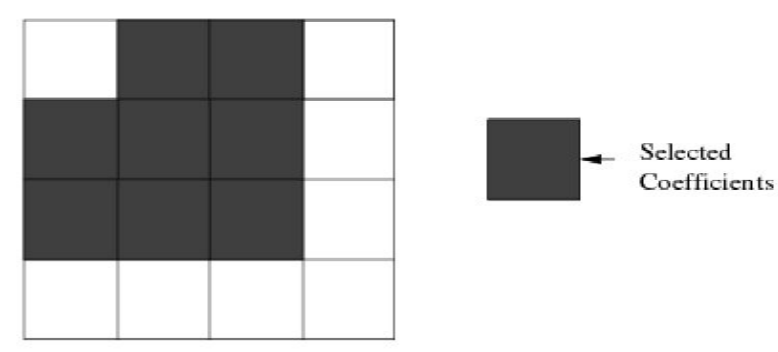

Figure 2: Coefficients selected for hiding watermark

into these selected coefficients as per the wavelet based algorithm. Thus each $4 \times 4$ block in the residual frame hides 8 bits from the watermark sequence. Multiple copies of the watermark sequence are embedded into the residual frames for robust recovery in case of losses. This data will be transmitted after post multiplication and quantization.

\subsection{Watermark Retrieval}

At the receiver, the video frames are reconstructed from the predicted frames and the residual data. In case of block losses due to transmission, the approximate values of those blocks can be recovered from the redundant watermark information in the residual frames. For this purpose, the recovered residual frame should be transformed through Int$\mathrm{DCT}$ and then from the 8 preselected locations of each $4 \times 4$ block, the watermark bits are recovered. The watermark bits are decided according to majority rule from the recovered stream and subsequently binary to decimal conversion and scale factor multiplication are performed. The entire structure of retrieval of the approximate band at the receiver is shown in Figure 3. From the retrieved LL2 band as

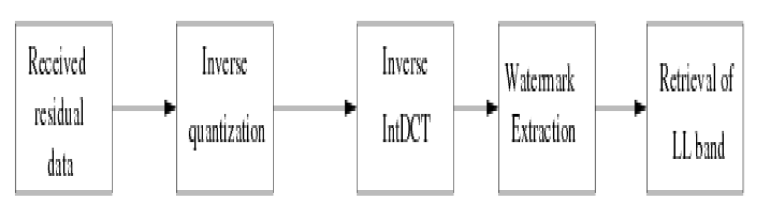

Figure 3: Watermark retrieval at the receiver

per the above method, the original frame is formed by performing the inverse wavelet transformation with all other high frequency coefficients set to zero. This gives the approximate information of the entire image and will be used for replacing error blocks.

\subsection{Concealment of Intra Coded Frames}

For intra coded frames, the block size for prediction could be $4 \times 4$ or $16 \times 16$ in case of H.264/AVC and after prediction the residual information is found out. IntDCT is performed on this residual frame and the watermark bits formed from the original frame is embedded into these transformed coefficients. After this stage post multiplication and quantization are performed. The transmitted data consists of both residual data with watermark embedded into it and the predicted I-frame.

The receiver receives the predicted frame as well as the residual data. The decoder detects the error locations. The residual data may contain $4 \times 4$ block losses and the predicted frame may contain $4 \times 4$ or $16 \times 16$ block losses or both. The block errors in the predicted frame are concealed from the approximate image obtained through watermarking. The residue information corresponding to these blocks are not utilized for further processing. Thus the predicted frame which is having its block errors replaced with their approximate values and the residual frame together give the intra coded frame. Since, the blocks in error are replaced by their approximate values, the high frequency information in these blocks are missing. This is retrieved through POCS. Thus the low frequency information as well as the high frequency information is restored on the intra coded frame. The complete block diagram of this process is given in Figure 4.

\subsection{Concealment of Inter Coded Frames}

The complete procedure involved in error concealment for inter coded video is shown in Figure 5. In case of inter prediction, the previously decoded frame is used as reference for motion vector estimation. The difference between the original frame and the motion compensated predicted frame forms the residual frame. Watermark bits are formed from the original frame as in the case of intra coding itself. These bits are embedded into the transform coefficients of the residual frame same as in the case of intra coded frames. Thus both motion vector information and residual information with watermark data are transmitted.

Errors occurred while transmission could be either due to 


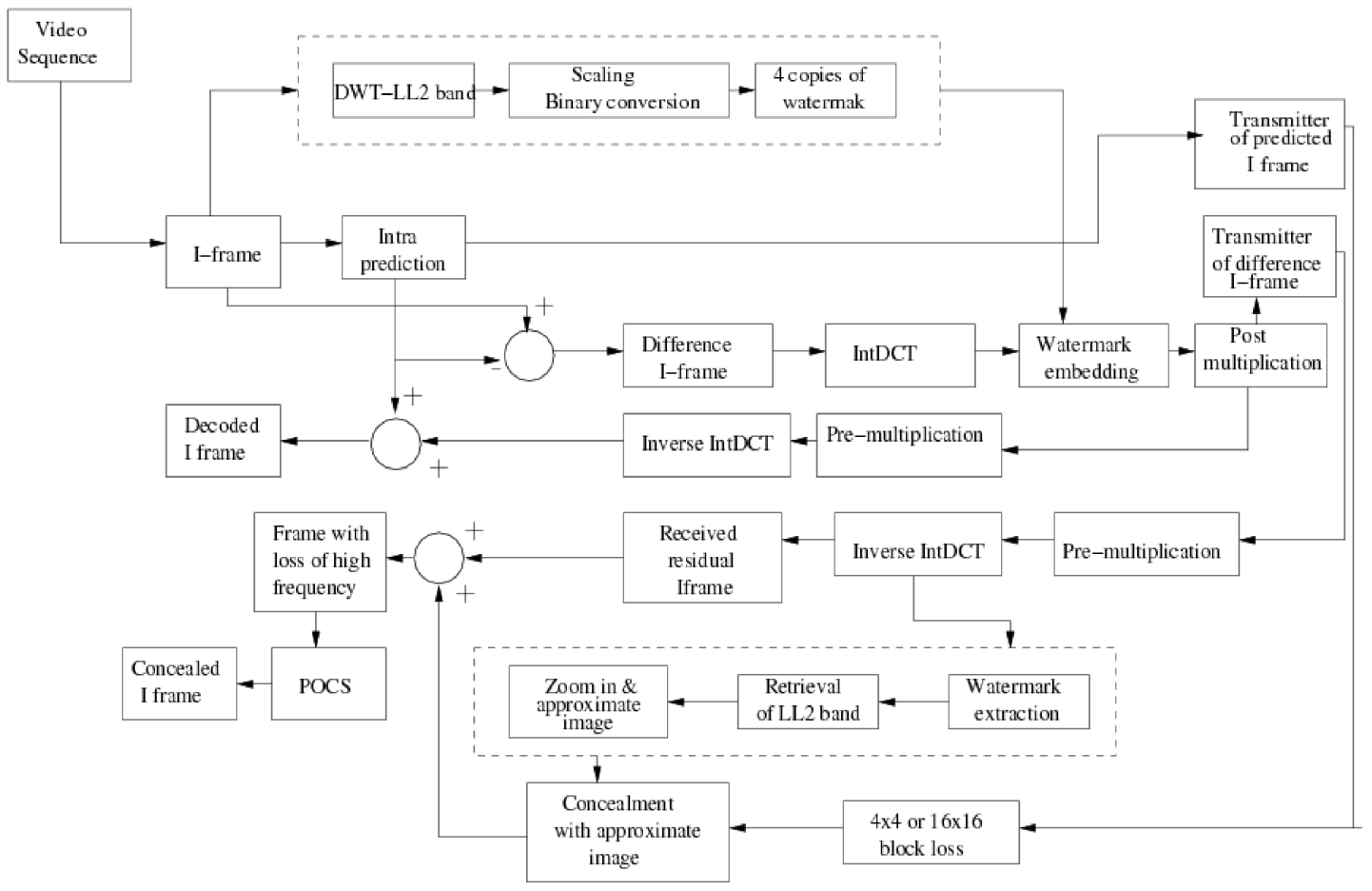

Figure 4: Error concealment in intra coded frames

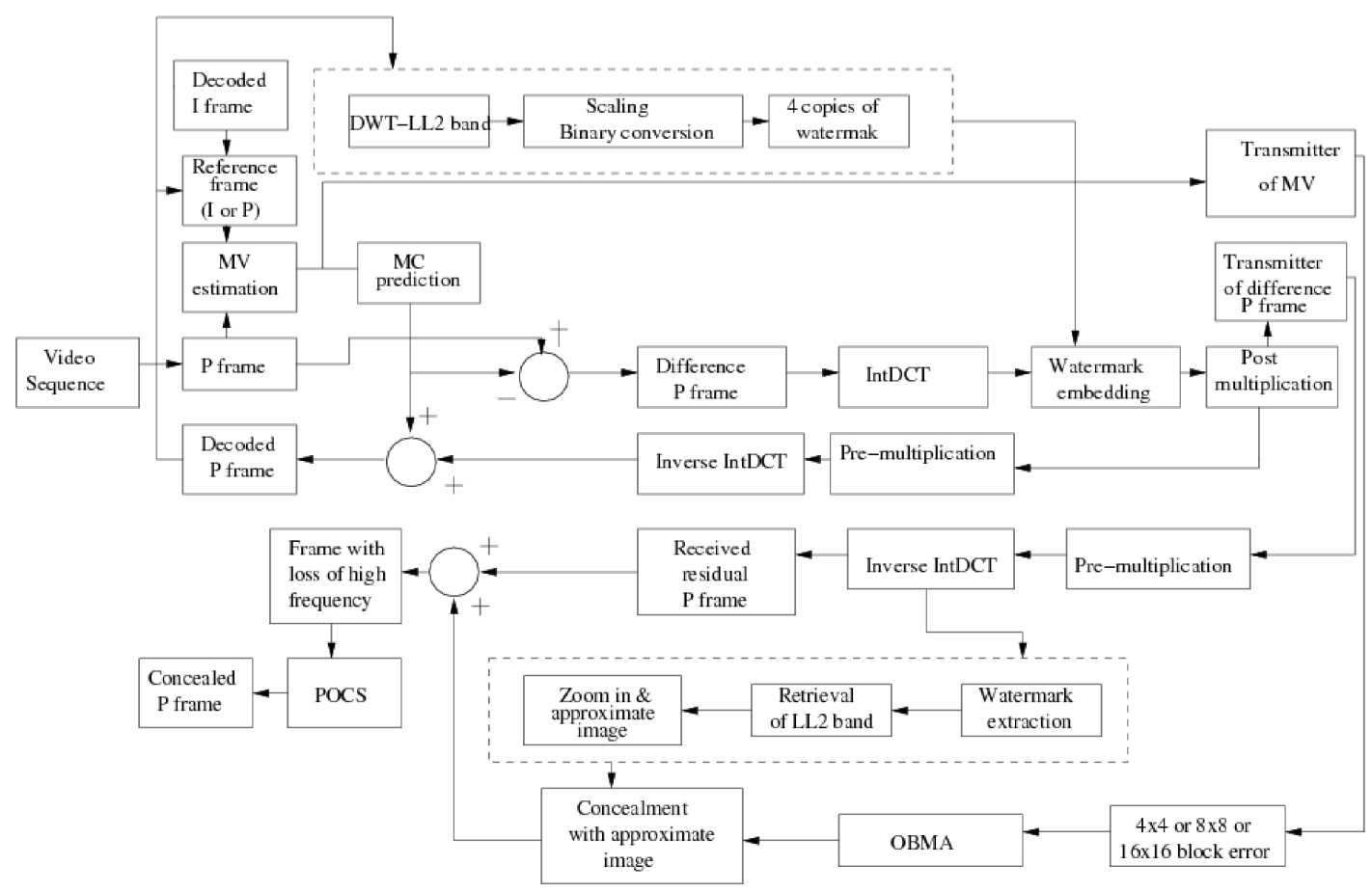

Figure 5: Error concealment in inter-coded frames 
loss of motion vector loss leading to block errors of different sizes or due to loss of residual data leading to $4 \times 4$ block error. For motion compensated prediction, previously decoded and concealed frames are used as reference.

- With less or no motion: In case of very less motion, the correlation of the macro-blocks of the error frame with those in the reference frame will be more than that with the approximate frame generated from the watermark bits. In case of uniform motion, the neighboring motion vectors will be highly correlated and any lost motion vector can be estimated accurately. Thus the lost block can be recovered. Hence BMA (Boundary Matching Algorithm) based concealment can be used. Moreover, if residual information is available for these blocks, it can enhance the results. In the proposed method, Overlapping Boundary Matching Algorithm (OBMA) is used to conceal errors in blocks which have less motion [3], [4]. From the available neighboring motion vectors the nature of motion is predicted. Both magnitude and direction of motion vectors are taken into consideration. The criteria for this concealment are

1. Motion Vectors of neighboring blocks are zero or very small

2. Motion Vectors are unidirectional with almost equal magnitude in their mean, median and individual values.

These concealed blocks through OBMA are added with residual data to recover the lost block.

- With large or complicated motion: Watermarking based error concealment is initiated only if the blocks have complex motion. In such cases, the lost blocks of the inter predicted frame are replaced by the corresponding values from the approximate image generated through watermark extraction. Then the residual frame and the predicted frame are combined to form the inter-coded frame. Still there could be error due to the loss of high frequency information. Moreover, some blocks in residual frame could themselves be in error. These errors can still cause visible distortion in video. In the next step of concealment POCS is applied. This stage is performed after calculating Sum of Absolute Difference (SAD) with the neighboring blocks and selecting block errors which are still predominant, which would finally help concealing all types of errors caused while transmission.

\subsection{Experimental Results}

Three different videos with different motion and texture characteristics are considered for testing the proposed method. Each frame of the video is of size $144 \times 176$. Thus there are $18 \times 22 \times 8=3168$ bits to be hidden in each frame. Total number of coefficients available for watermark hiding are $\frac{144 \times 176 \times 8}{4 \times 4}=12672$. Thus $\frac{12672}{3168}=4$ copies of the watermark are embedded in each frame. Random block errors were introduced to the frames to simulate channel losses. Three test videos were used for testing the proposed method with different percentage block errors introduced into them. The results have been compared with BMA based error concealment to demonstrate the improvement of the proposed method. The proposed method shows very good concealment of block errors irrespective of the type of

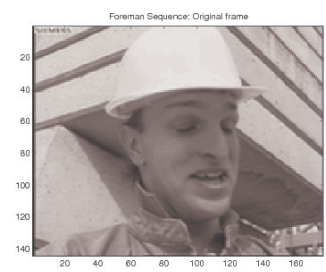

(a)

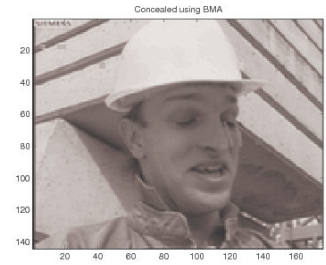

(c)

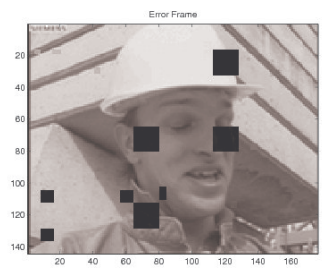

(b)

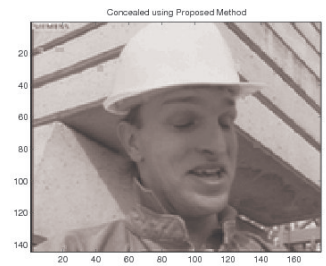

(d)
Figure 6: a. Original frame, b. Error frame c. Error Concealed frames-BMA d. Error Concealed framesProposed method

motion, whether low or high, whereas, BMA method shows serious distortion in case of large motion.

The Table 1 shows the PSNR and SSIM index of the videos selected under different loss rate. The percentage error shown is the percentage of total frames in error. In each of the error frame $8 \%$ to $10 \%$ macro-blocks are in error. The performance of the proposed method is much superior with higher amount of block error. Table 2 shows the results of concealment with a different loss rates in the residual frame. Percentage error shows the percentage of residual frames in error and in each error frame $8 \%$ to $10 \% 4 \times 4$ macro-blocks are in error. Even when there is loss of residual data, the proposed method gives superior performance as POCS in the subsequent stage accounts for the high frequency components.

Figure 7 shows the PSNR and SSIM index for Foreman sequence. The plots are obtained for 100 frames of Foreman sequence with 45 inter-coded erroneous frames and 28 residual erroneous frames. The advantage of the proposed method is very obvious in case of errors in those blocks which have large or complicated motion. Figure 8 shows the frame numbers in error and the number of lost blocks.

\section{CONCLUSION}

Transmission of images through noisy channels often leads to loss of blocks of data. The proposed scheme makes used of the advantage of watermarking based scheme to reduce the computational complexity involved in POCS. Watermarking based scheme restores these lost data through the retrieval of a watermark embedded in the transmitted image.

Temporal concealment schemes need the motion vectors to be estimated with a satisfactory degree of accuracy, which is limited by the available information from neighboring blocks. The proposed technique for video error concealment can overcome such limitations. It exploits the neighboring Motion Vector (MV) information, if a good estimate of lost $\mathrm{MV}$ is possible. Else watermarking based scheme does the 


\begin{tabular}{|c|c|c|c|c|c|c|c|}
\hline $\begin{array}{c}\text { Test } \\
\text { Sequence }\end{array}$ & $\begin{array}{c}\text { \% Frame } \\
\text { error }\end{array}$ & $\begin{array}{c}\text { PSNR } \\
\text { Error }\end{array}$ & $\begin{array}{c}\text { PSNR } \\
\text { BMA }\end{array}$ & $\begin{array}{c}\text { PSNR } \\
\text { Proposed }\end{array}$ & $\begin{array}{c}\text { SSIM } \\
\text { Error }\end{array}$ & $\begin{array}{c}\text { SSIM } \\
\text { BMA }\end{array}$ & $\begin{array}{c}\text { SSIM } \\
\text { Proposed }\end{array}$ \\
\hline \multirow{3}{*}{ Coastguard } & $35 \%$ & 24.6481 & 42.0484 & 42.1250 & 0.9128 & 0.9938 & 0.9947 \\
& $60 \%$ & 22.5091 & 40.8268 & 41.7268 & 0.8724 & 0.9907 & 0.9924 \\
& $85 \%$ & 19.2089 & 34.5724 & 36.3784 & 0.7556 & 0.9781 & 0.9871 \\
\hline \multirow{3}{*}{ Foreman } & $35 \%$ & 24.2901 & 45.5624 & 45.7826 & 0.8916 & 0.9953 & 0.9959 \\
& $60 \%$ & 20.8907 & 44.1817 & 44.9672 & 0.8340 & 0.9935 & 0.9939 \\
& $85 \%$ & 18.3397 & 41.0509 & 42.1575 & 0.7199 & 0.9871 & 0.9893 \\
\hline \multirow{3}{*}{ Husky } & $35 \%$ & 26.0410 & 37.3507 & 37.7357 & 0.9300 & 0.9873 & 0.9886 \\
& $60 \%$ & 23.8261 & 34.9833 & 35.6249 & 0.8977 & 0.9817 & 0.9824 \\
& $85 \%$ & 20.9007 & 31.3442 & 32.9362 & 0.8037 & 0.9511 & 0.9641 \\
\hline
\end{tabular}

Table 1: PSNR and SSIM after concealment of block errors

\begin{tabular}{|c|c|c|c|c|c|c|c|}
\hline $\begin{array}{c}\text { Test } \\
\text { Sequence }\end{array}$ & $\begin{array}{c}\text { \% Frame } \\
\text { error }\end{array}$ & $\begin{array}{c}\text { PSNR } \\
\text { Error }\end{array}$ & $\begin{array}{c}\text { PSNR } \\
\text { BMA }\end{array}$ & $\begin{array}{c}\text { PSNR } \\
\text { Proposed }\end{array}$ & $\begin{array}{c}\text { SSIM } \\
\text { Error }\end{array}$ & $\begin{array}{c}\text { SSIM } \\
\text { BMA }\end{array}$ & $\begin{array}{c}\text { SSIM } \\
\text { Proposed }\end{array}$ \\
\hline \multirow{3}{*}{ Coastguard } & $45 \%$ & 27.6481 & 42.0484 & 42.1250 & 0.9198 & 0.9938 & 0.9947 \\
& $50 \%$ & 26.6545 & 41.3428 & 41.6190 & 0.9195 & 0.9929 & 0.9931 \\
& $55 \%$ & 24.8672 & 41.3200 & 41.4033 & 0.9166 & 0.9922 & 0.9924 \\
& $85 \%$ & 18.6883 & 34.2308 & 35.6583 & 0.7421 & 0.9753 & 0.9788 \\
\hline \multirow{5}{*}{ Foreman } & $45 \%$ & 27.4241 & 45.9838 & 46.1537 & 0.9196 & 0.9954 & 0.9959 \\
& $50 \%$ & 25.4271 & 45.5904 & 45.6811 & 0.8984 & 0.9953 & 0.9956 \\
& $55 \%$ & 24.2901 & 45.5624 & 45.7826 & 0.8916 & 0.9949 & 0.9950 \\
& $85 \%$ & 16.8263 & 37.3922 & 38.5102 & 0.6779 & 0.9757 & 0.9808 \\
\hline \multirow{6}{*}{ Husky } & $45 \%$ & 27.0410 & 37.7507 & 37.9357 & 0.9370 & 0.9905 & 0.9906 \\
& $50 \%$ & 26.9426 & 37.6804 & 37.8464 & 0.9365 & 0.9903 & 0.9904 \\
& $55 \%$ & 26.1894 & 37.0973 & 37.3719 & 0.9362 & 0.9896 & 0.9902 \\
& $85 \%$ & 20.6790 & 31.1535 & 32.3385 & 0.8084 & 0.9537 & 0.9625 \\
\hline
\end{tabular}

Table 2: PSNR and SSIM after concealment of block errors in residual frames
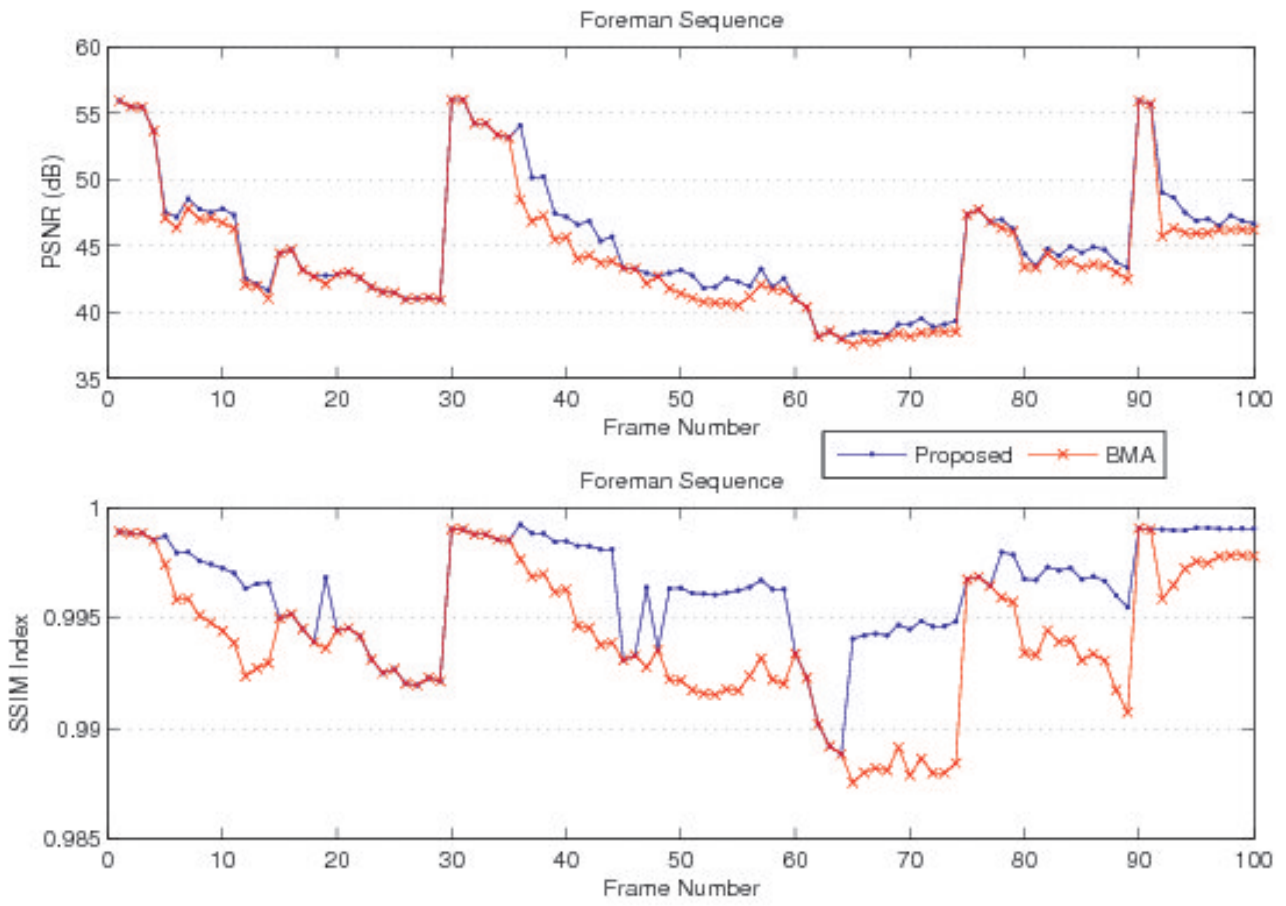

Figure 7: PSNR ans SSIM by proposed method and BMA method 

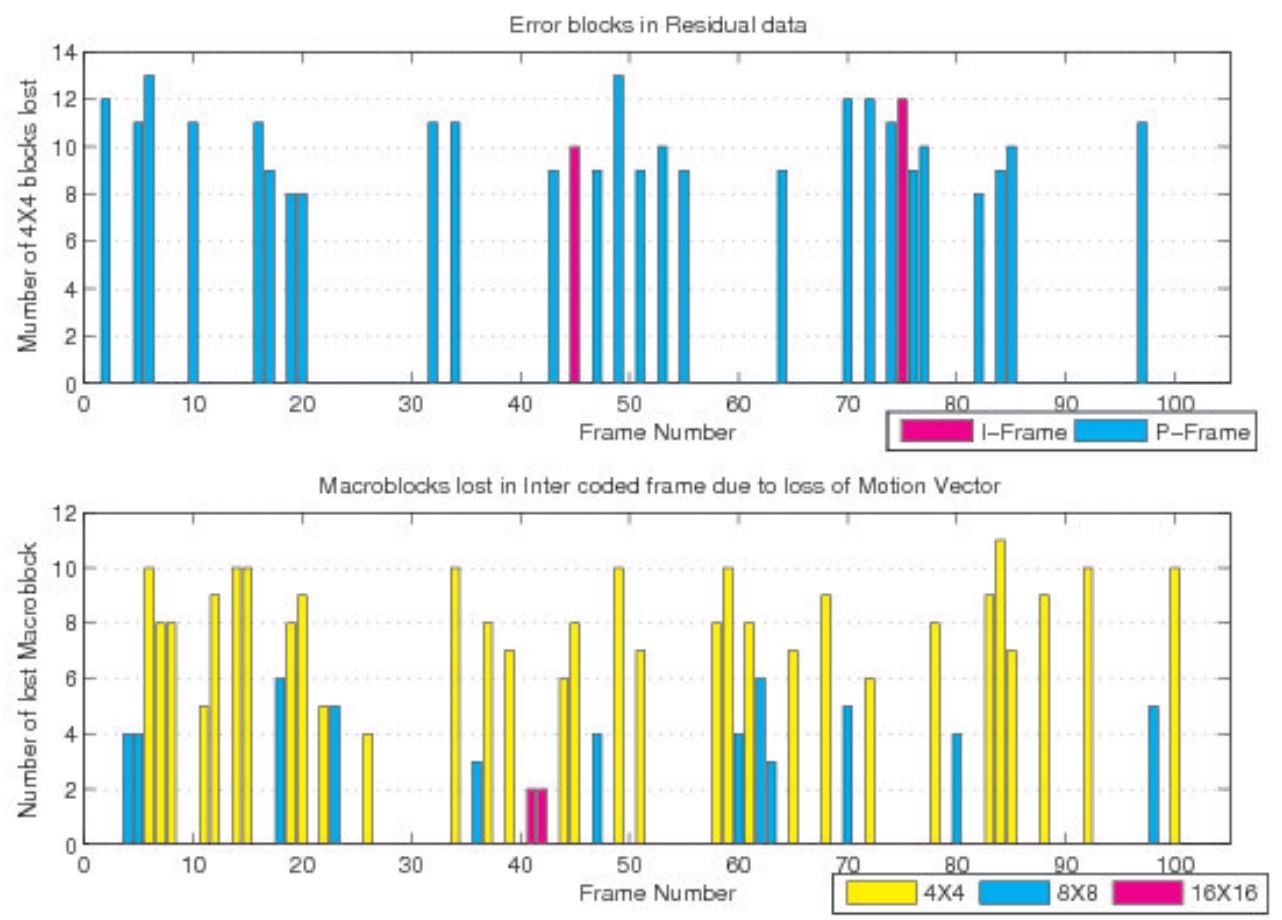

Figure 8: Error blocks in residual data and macro-blocks lost

spatial concealment of lost block. The proposed method for error concealment gives noticeably superior performance when there is complex motion in the video. Otherwise, OBMA based technique is sufficient for concealment. In case of large number of erroneous blocks, watermark retrieved for concealing the errors may itself be in error. But POCS can detect these errors and conceal in successive steps. Hence this scheme is suitable for both intra coded and inter coded frames with different types of motion and varied error environment.

\section{REFERENCES}

[1] Adsumilli, B. C. B., Farias, M. C. Q., Mitra, S. K., Carli, M., Nov. 2005. A robust error concealment technique using data hiding for image and video transmission over lossy channels. IEEE Transaction on Circuit and Systems for Video Technology 15 (11), 1394-1406.

[2] Fu, F., Lin, X., Xu, L., Dec. 2004. Fast intra prediction algorithm in h.264/avc. ICSP Proceedings, 1191-1194.

[3] Kim, D., Jeong, S. Y. J., Sep. 2005. A new temporal error concealment method for h.264 using adaptive block sizes. IEEE International Conference on Image Processing, 928-931.

[4] Lam, W. M., Reibman, A., B.Liu, Apr. 1993. Recovery of lost or erroneously received motion vectors. IEEE Int. Conf. on Acoustics, Speech and Signal Processing 5, 417-412.

[5] Luthara, A. K., Sullivan, G. J., Jul. 2003. Introduction to the special issue on the h.264/avc video coding standard. IEEE Trans. Circuits and
Systems for Video Technology 13 (7), 557-559.

[6] M., J., Merchant, S. N., Desai, U. B., G., A., M., A., P., S., J., S., Dec. 2006. Error concealment using digital watermarking. Asia Pacific Conference on Circuits and Systems, $1713-1716$.

[7] Nayak, C. K., M., J., Merchant, S. N., Desai, U. B., Dec. 2007. Projection onto convex sets with watermarking for error concealment. Int. Conf. Pattern Recognition and Machine Intelligence, LNCS 4815/2007, 119-127.

[8] Sullivan, G. J., Wiegand, T., Jan. 2005. Video compression- from concepts to the h.264/avc standard. Proceedings of IEEE 93 (1), 18-31.

[9] Sun, H., Kwok, W., Apr. 1995. Concealment of damaged block transform coded images using projections onto convex sets. IEEE Transaction on Image Processing 4 (4), 470-477.

[10] T.Wiegand, Suliivan, G. J., Bjontegaard, G., Luthra, A., Jul. 2003. Overview of the h.264/avc video coding standard. IEEE Trans. Circuits and Systems for Video Technology 13 (7), 560-576.

[11] Wang, Y., Zhu, Q., May 1998. Error control and concealment for video communications. IEEE Trans. 86, 974-995.

[12] Wang, Z., Bovik, A. C., Sheikh, R., Simoncelli, E. P., Apr. 2004. Image quality assessment: From error visibility to structural similarity. IEEE Transaction on Image Processing 13 (4), 600-612. 